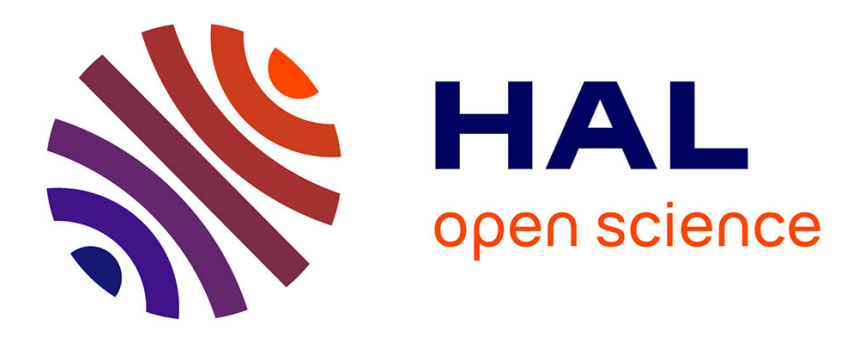

\title{
Direction et distance d'analyse à la sonde atomique
}

\author{
D. Blavette, J.M. Sarrau, A. Bostel, J. Gallot
}

\section{To cite this version:}

D. Blavette, J.M. Sarrau, A. Bostel, J. Gallot. Direction et distance d'analyse à la sonde atomique. Revue de Physique Appliquée, 1982, 17 (7), pp.435-440. 10.1051/rphysap:01982001707043500 . jpa00245019

\section{HAL Id: jpa-00245019 https://hal.science/jpa-00245019}

Submitted on 1 Jan 1982

HAL is a multi-disciplinary open access archive for the deposit and dissemination of scientific research documents, whether they are published or not. The documents may come from teaching and research institutions in France or abroad, or from public or private research centers.
L'archive ouverte pluridisciplinaire HAL, est destinée au dépôt et à la diffusion de documents scientifiques de niveau recherche, publiés ou non, émanant des établissements d'enseignement et de recherche français ou étrangers, des laboratoires publics ou privés. 
Classification

Physics Abstracts

07.80

\title{
Direction et distance d'analyse à la sonde atomique
}

\author{
D. Blavette, J. M. Sarrau, A. Bostel et J. Gallot \\ Groupe de Métallurgie Physique, ERA 258, UER des Sciences et Techniques, \\ B.P. 67, 76130 Mont Saint Aignan, France
}

(Reçu le 18 janvier 1982, révisé le 14 avril 1982, accepté le 19 avril 1982)

\begin{abstract}
Résumé. - Les résultats d'analyse à la sonde atomique se présentent sous la forme d'une liste d'arrivée des atomes qui correspond à l'investigation en profondeur de l'échantillon. Pour une pleine exploitation des résultats, en particulier dans les profils de composition, cette liste doit être reliée au réseau réel de l'échantillon.

A partir d'un modèle représentant la forme des échantillons, la direction et la surface d'analyse sont définies en tout site d'investigation. La profondeur d'analyse est ensuite exprimée en fonction du nombre de plans évaporés. Une expression du volume analysé est proposée et conduit à une relation donnant la profondeur d'analyse en fonction du nombre d'atomes reçus. Enfin une constante d'expérimentation est introduite et montre la contribution des différents facteurs mis en jeu.
\end{abstract}

\begin{abstract}
The atom-probe results of analysis can be represented by a sequencial list of evaporated atoms corresponding to the sample investigation in depth. For a good exploitation, in particular for composition profiles, this list should be related to the real lattice of the sample.

From an emitter shape model, the direction and the surface analysis are defined for every investigation site. The analysis depth is determined from the number of evaporated planes. An expression of the analysed volume is given and leads to a relation between the analysis depth and the number of detected atoms. An experimental constant, which shows the contribution of the different factors, is then introduced.
\end{abstract}

La sonde atomique associée à un microscope ionique trouve une de ses principales applications dans l'étude des alliages $[1,2,3]$. La grande résolution spatiale de l'analyse tant en profondeur qu'en surface de l'échantillon, en fait un outil d'analyse particulièrement efficace. La sonde permet de caractériser la distribution des différents types de solutés dans le volume, mais aussi au voisinage d'un interface ou d'un joint de grain $[4,5,6,7]$.

L'échantillon est évaporé par effet de champ atome par atome. Une partie de ces atomes évaporés est recueillie sur un détecteur, dont l'image sur la surface de l'échantillon découpe une zone d'analyse. Les ions ainsi détectés sont identifiés par spectrométrie de masse à temps de vol, et constituent une « liste d'arrivée " d'ions. Pour exploiter toute l'information, la relation entre cette liste et le réseau réel de l'échantillon doit être déterminée dans tous les cas de figure. Ceci pose le problème de la détermination de la taille de la surface analysée, de la direction analysée, du site cristallographique de la surface concernée.

La détermination précise de ces données essentielles permet d'exploiter utilement la liste d'arrivée; en particulier pour obtenir des profils de composition.
Dans ce cas, il est important de relier le nombre d'atomes (détectés sous forme d'ions) à la profondeur d'analyse. De plus, la direction d'analyse, sauf cas particulier, n'est pas normale à la surface mais dépend du site analysé ; la relation $d=n d_{h k l}$ donnant la profondeur analysée à partir du nombre de plans évaporés n'est vérifiée que si le site d'analyse est proche de l'axe de l'échantillon.

Un modèle simple de la forme des échantillons permet de mettre en évidence la dépendance de la direction, de la surface et du volume d'analyse avec le site d'investigation. Dans cette étude, les effets dus à des variations de courbure locale provenant par exemple de la présence de deux phases, ne sont pas considérés.

1. Analyse. - Une région déterminée de l'échantillon est amenée face au détecteur à l'aide d'une tête goniométrique (Fig. 1) [8]. En inclinant l'axe de l'échantillon d'un angle $\psi$, puis en tournant cet échantillon autour de l'axe du spectromètre, on sélectionne un site choisi de l'échantillon.

L'échantillon est assimilé à un tronc de cône terminé par une calotte sphérique ("Core ») $[9,10,11]$. Les trajectoires des ions métalliques évaporés tendent 


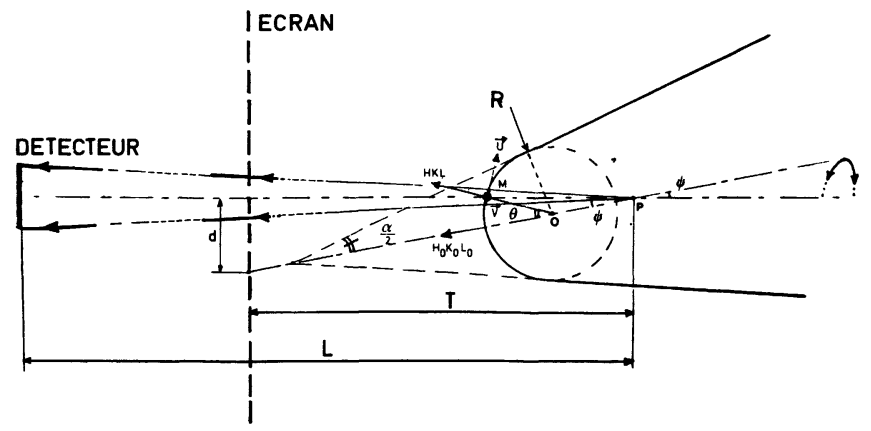

Fig. 1. - Surface d'analyse et site d'investigation.

[Analysis area and investigation area.]

asymptotiquement vers des directions qui se coupent en un point $\mathrm{P}$ "de projection " lié à l'échantillon. Si $R$ est le rayon de courbure de l'échantillon, le point $P$ est défini par : $\mathrm{OP}=m R[12,13,14]$.

La projection est stéréographique lorsque $m=1$. Si $\theta$ est l'angle entre les directions $(H K L)$ et $\left(H_{0} K_{0} L_{0}\right)$; l'échantillon est orienté de l'angle $\psi$ nécessaire pour localiser l'analyse sur le site $(h k l)$ tel que :

$$
\operatorname{tg} \psi=\sin \theta /(m+\cos \theta)
$$

Parmi les trois variables $m, \theta$ et $\psi$, seules deux d'entre elles sont nécessaires pour décrire complètement une direction d'analyse : par exemple $m$ et $\theta$, ou bien $\psi$ et $\theta$. $\psi$ correspond à l'angle de visée et peut être déterminé à l'aide de l'image ionique par la relation :

$$
\operatorname{tg} \psi=d / T \quad \text { (Fig. 1). }
$$

A partir de $\theta$ et $\psi$, données expérimentales, $m$ est calculé :

$$
m=\sin (\theta-\psi) / \sin \psi .
$$

Dans les calculs présentés, seuls $m$ et $\theta$ apparaissent ; puisque $m$ et $\psi$ sont des données équivalentes.

1.1 SURFACE ANAlyséE. - La distance $L$ pointedétecteur est grande vis-à-vis du rayon de courbure $R$. L'image du détecteur rapportée sur l'échantillon est une surface définie par deux vecteurs $\mathbf{u}$ et $\mathbf{v}$ orthogonaux, appartenant au plan tangent à la sphère. De plus, u est contenu dans le plan formé par l'axe du spectromètre et l'axe de l'échantillon. Les deux grandissements $G_{u}$ et $G_{v}$ sont définis par :

$$
G_{u}=L \cdot \cos (\theta-\psi) / \mathrm{PM} \text { et } G_{v}=L / \mathrm{PM} .
$$

La surface analysée, de forme elliptique, se déduit de la surface du détecteur par une projection définie par :

$$
S_{\mathrm{a}}=S_{\mathrm{D}} / G_{u} \cdot G_{v} .
$$

Si $D$ est le diamètre du détecteur, le petit axe et le grand axe de l'ellipse s'expriment de la manière sui- vante :

$$
d_{v}=D / G_{v} \quad \text { et } \quad d_{u}=D / G_{u} .
$$

La distance PM définissant les grandissements $G_{u}$ et $G_{v}$ est :

$$
\mathrm{PM}=R \cdot\left(1+2 m \cos \theta+m^{2}\right)^{1 / 2} .
$$

Le facteur $\cos (\theta-\psi)$ faisant intervenir l'angle $\psi$ peut être mis sous la forme :

$$
\cos (\theta-\psi)=(1+m \cos \theta) /\left(1+2 m \cos \theta+m^{2}\right)^{1 / 2} .
$$

L'existence de deux grandissements $G_{u}$ et $G_{v}$ liés entre eux par ce facteur, montre que l'image du réseau réel de l'échantillon subit une distorsion d'autant plus marquée que $\theta$ est grand.

Soit $G$ le grandissement sur l'axe de la pointe $\left(H_{0} K_{0} L_{0}\right)$; en posant

$\gamma(m, \theta)=\left(1+2 m \cos \theta+m^{2}\right)^{3 / 2} /(m+1)^{2} \cdot(1+m \cos \theta)$.

La surface analysée est alors :

$$
S_{\mathrm{a}}=S_{\mathrm{D}} \cdot \gamma(m, \theta) / G^{2} .
$$

Le grandissement sur l'axe de l'échantillon est donné par :

$$
G=L /(m+1) \cdot R .
$$

La surface analysée est proportionnelle au carré du rayon de courbure de l'échantillon. Ces relations restent valables tant que $\theta$ est inférieur à un angle limite $\theta_{\mathbf{e}}$ tel que

$$
\theta_{\mathrm{e}}+\alpha / 2=\pi / 2 .
$$

La figure 2 montre l'aspect de la fonction $\gamma(\theta)$ pour différentes valeurs de $m$. Pour $\theta<30^{\circ}$ la surface d'analyse reste peu différente de $S_{\mathrm{D}} / G^{2}$.

1.2 Direction efFective D'ANALYSE. - Le modèle d'échantillon étant un tronc de cône terminé par une calotte sphérique, l'évaporation couche par couche des atomes entraîne une augmentation du rayon de

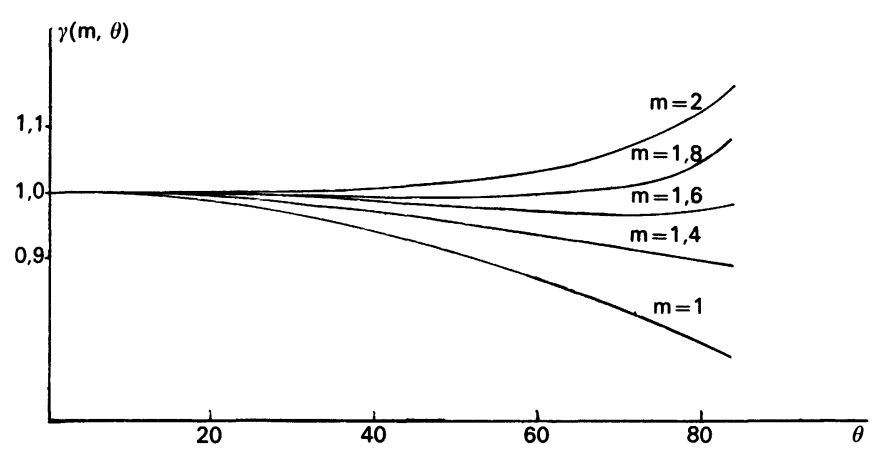

Fig. 2. - Fonction $\gamma(m, \theta)$ pour différentes valeurs de $m$. $[\gamma(m, \theta)$ function for different values of $m$. $]$ 
courbure. Le phénomène est vérifié expérimentalement par la nécessité d'augmenter le potentiel appliqué pour maintenir le champ d'évaporation. La figure 3 montre l'évolution théorique d'un tel modèle. La distance $(L)$ pointe-détecteur est grande vis-à-vis du rayon de courbure. La zone d'analyse reste située au cours de l'évaporation sur le site $(H, K, L)$. Le parallaxe $p$ est négligé puisqu'il est très faible vis-à-vis du diamètre du détecteur. Dans ces conditions, la direction d'analyse n'est plus normale à la surface d'analyse, mais fait un angle $\phi$ avec l'axe de l'échantillon, tel que :

$$
\operatorname{tg} \phi=\sin \theta \cdot \sin \alpha / 2 /(1-\sin \alpha / 2 \cdot \cos \theta) .
$$

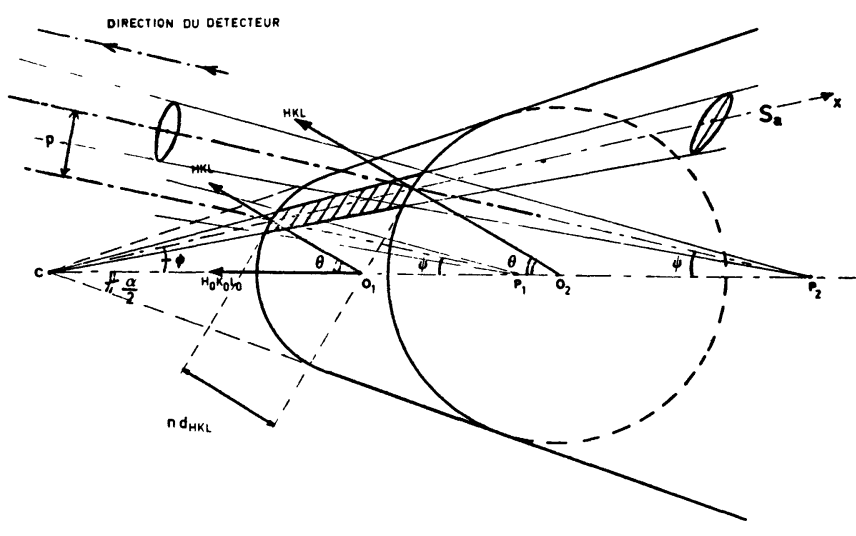

Fig. 3. - Evolution théorique de l'échantillon. Distance et volume analysés.

[Theoretical evolution of the sample shape. Distance and volume of analysis.]

L'angle formé par la normale au plan et la direction d'analyse est $\theta+\phi$. Toutes les surfaces d'analyse sont orientées d'un angle $\theta(H, K, L)$ constant. L'expression de $\phi$ montre que si l'angle de cône reste constant au cours de l'analyse, la direction d'analyse est une droite qui fait un angle $\phi$ avec l'axe de la pointe. $\phi$ ne dépend pas de la position du point de projection $P$. La figure 4 montre l'aspect de la fonction $\phi(\theta)$ pour différentes valeurs de $\alpha$. Lorsque $\theta$ est faible, $\phi$ est peu différent de $\theta \cdot \sin \alpha / 2$.

1.3 Distance ANAlYSÉE. - La distance analysée suivant l'axe $c x$, correspondant à une variation infinitésimale $\mathrm{d} R$ du rayon de courbure est :

$$
\mathrm{d} x=\mathrm{d} R / k_{\alpha} \cdot k_{\theta}
$$

avec

$$
k_{\alpha}=\sin \alpha / 2 /(1-\sin \alpha / 2)
$$

et

$$
k_{\theta}=1 /\left(1+\left(2 \cdot k_{\alpha} \cdot \sin \theta / 2\right)^{2} / \sin \alpha / 2\right)^{1 / 2} .
$$

La distance analysée $d_{\mathrm{an}}$ est proportionnelle à la

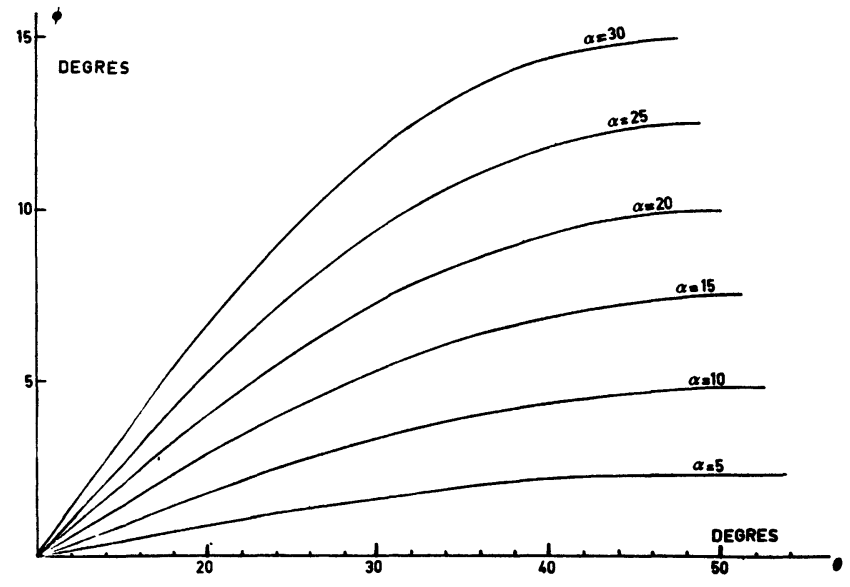

Fig. 4. - Direction d'analyse en fonction de $\theta$ pour différents angles de cône.

[Analysis direction versus $\theta$ for different shank angles.]

variation $R$ du rayon de courbure :

$$
d_{\mathrm{an}}=\Delta R / k_{\alpha} \cdot k_{\theta} .
$$

Lorsque l'analyse est faite sur l'axe de la pointe, $k_{\theta}=1$ par suite $d_{\mathrm{an}}=\Delta R / k_{\alpha}$. Pour de petits angles de cône, $k_{\theta}$ est peu différent de 1 , et $k_{\alpha}$ de $\alpha / 2$.

Relation avec le nombre de plans évaporés. - En choisissant le site d'analyse sur un bord de plan près d'un pôle dont les indices de Miller sont connus, il est possible de séparer la liste d'arrivée des ions par plan évaporé. Si la largeur de la terrasse $l$ est supérieure aux dimensions de la surface analysée, la courbe représentant le nombre d'atomes reçus en fonction du nombre d'impulsions d'évaporation appliquées à l'échantillon présente une suite de marches. Chaque marche correspond à un plan évaporé. La largeur de la $j$-ième terrasse comptée à partir du centre du pôle vaut approximativement :

$$
l=\left(2 R \cdot d_{H K L} / j\right)^{1 / 2} .
$$

Si le nombre de plans évaporés est connu expérimentalement, la distance analysée peut être reliée à la distance $d_{h k l}$ entre ces plans. L'angle formé par la normale aux plans et la direction d'analyse est $\theta+\phi$, de sorte que :

$$
d_{\mathrm{an}}=n \cdot d_{H K L} / \cos (\theta+\phi) \quad \text { (Fig. 3) }
$$

1.4 Volume ANALYSÉ. - Les surfaces analysées se déduisent les unes des autres par une homothétie; elles sont proportionnelles au carré du rayon de courbure de l'échantillon. Ce dernier étant lui-même proportionnel à la distance, le volume analysé est un tronc de cône enveloppant toutes les surfaces analysées.

Le volume $\mathrm{d} v$ correspondant à une petite variation $\mathrm{d} R$ du rayon de courbure, et associé à la surface d'analyse $S_{\mathrm{a}}$ vaut :

$$
\mathrm{d} v=S_{\mathrm{a}} \cdot \cos (\theta+\phi) \cdot \mathrm{d} x .
$$


En remplaçant $S_{\mathrm{a}}$ et dx par leurs expressions données en (4) et (8), en intégrant entre $R_{0}-\Delta R / 2$ et $R_{0}+\Delta R / 2$ on obtient :

$v=\int_{R_{0}-\Delta R / 2}^{R_{0}+\Delta R / 2}\left(S_{\mathrm{D}} / L^{2}\right) \cdot(m+1)^{2} \cdot \gamma(m, \theta) \times$
$\times \cos (\theta+\phi) \cdot\left(k_{\alpha} \cdot k_{\theta}\right)^{-1} \cdot R^{2} \mathrm{~d} R$

$R_{0}$ est le rayon de courbure moyen de l'échantillon. $\Delta R$ est suffisamment petit pour que $m$ et $\alpha$ soient considérés comme constants. Dans ces conditions, le volume s'écrit :

$$
\begin{aligned}
v=\left(S_{\mathrm{D}} R_{0}^{2} / L^{2}\right) \cdot(m+1)^{2} \cdot \gamma(m, v) \cdot \cos (\theta+\phi) \times \\
\times\left(\Delta R / k_{\alpha} k_{\theta}\right) \cdot\left|1+\left(\Delta R / R_{0}\right)^{2} / 12\right| .
\end{aligned}
$$

Si $\Delta R<R_{0}$ :

$v=\left(S_{\mathrm{D}} R_{0}^{2} / L^{2}\right) \cdot(m+1)^{2} \cdot \gamma(m, \theta) \cdot \cos (\theta+\phi) \cdot \Delta R / k_{\alpha} \cdot k_{\theta}$.

En introduisant la distance analysée, le volume se met sous la forme :

$$
v=\left(S_{\mathrm{D}} \cdot R_{0}^{2} \cdot(m+1)^{2} / L^{2}\right) \cdot \gamma(m, \theta) \cdot \cos (\theta+\phi) \cdot d_{\mathrm{an}} .
$$

A $R_{0}$ correspond une surface moyenne d'analyse $S_{\mathrm{a} 0}$ avec $S_{\mathrm{a} 0}=S_{\mathrm{D}} \cdot \gamma(m, \theta) / G_{0}^{2}$; où $G_{0}$ est le grandissement moyen associé : $G_{0}=\frac{L}{(m+1) R_{0}}$. Dans ces conditions, le volume analysé devient :

$$
v=S_{\mathrm{a} 0} \cdot d_{\mathrm{an}} \cdot \cos (\theta+\phi) .
$$

Dans le cas particulier d'une analyse sur l'axe de l'échantillon, le volume analysé est le produit de la surface analysée par la distance analysée avec :

$$
S_{\mathrm{a} 0}(\theta=0)=S_{\mathrm{D}} / G_{0}^{2} .
$$

2. Applications. - Les données expérimentales sont d'une part le nombre $N$ d'atomes reçus, le potentiel moyen d'évaporation $V_{0}$, la variation de potentiel associée $\Delta V$, et d'autre part l'angle de visée $\psi$ et le pôle cristallographique d'analyse $(\theta)$. Le potentiel d'évaporation dépend du rayon de courbure de l'échantillon $[15,16]$ par :

$$
V=E \beta R \text {. }
$$

Le nombre $N$ d'atomes reçus est relié statistiquement au volume évaporé par le rendement $Q$ du détecteur :

$$
N \cdot v_{\mathrm{at}}=Q \cdot v,
$$

où $v_{\text {at }}$ est le volume atomique moyen.

2.1 RELATION ENTRE DISTANCE ET NOMBRE D'ATOMES REÇUS. - Un profil de composition est un graphe sur lequel on porte le nombre cumulé d'atomes de type A reçus en fonction du nombre total $N$ d'atomes reçus. L'expression (17) permet d'exprimer la profondeur d'analyse en fonction du nombre d'atomes reçus; et donc de porter une échelle des distances sur le profil de composition. Avec les expressions (17) et (14) :

$$
\begin{aligned}
d_{\mathrm{an}}=\left|L^{2} / S_{\mathrm{D}} Q\right| \cdot \mid(E \beta)^{2} \cdot v_{\mathrm{at}} /(m & +1)^{2}|\cdot| 1 / \gamma(m, \theta) \times \\
& \times \cos (\theta+\phi) \mid \cdot N / V_{0}^{2} .
\end{aligned}
$$

Les trois facteurs entre crochets dépendent respectivement des caractéristiques de la sonde, de l'échantillon, de l'orientation.

$N$ est le nombre d'atomes reçus entre les potentiels $V_{1}$ et $V_{2}$, tels que $V_{0}=\left(V_{1}+V_{2}\right) / 2$. La distance analysée est proportionnelle à $N / V_{0}^{2}$.

2.2 RENDEMENT EFFECTIF. - En utilisant l'expression (13) du volume analysé, et en lui associant la variation correspondante du potentiel d'évaporation $(\Delta V=E \beta \Delta R)$, l'expression $N v_{\text {at }}=Q \cdot v$ (17) devient, en groupant les termes liés à la sonde et à l'échantillon :

$$
\begin{aligned}
\mid S_{\mathrm{D}} Q \cdot(m+1)^{2} / L^{2} \cdot v_{\mathrm{at}} \cdot & k_{\alpha} \cdot(E \beta)^{3}|\cdot| \gamma(m, \theta) \times \\
& \times \cos (\theta+\phi) / k_{\theta} \mid=N /\left(V_{0}^{2} \Delta V\right) .
\end{aligned}
$$

Soient :

$$
\Gamma(m, \theta)=\gamma(m, \theta) \cdot \cos (\theta+\phi) / k_{\theta}
$$

et

$$
A=S_{\mathrm{D}} Q \cdot(m+1)^{2} / L^{2} \cdot v_{\mathrm{at}} \cdot k_{\alpha} \cdot(E \beta)^{3} .
$$

L'expression précédente peut se mettre sous la forme :

$$
A_{\theta}=A . \Gamma(m, \theta) \text { avec } A_{\theta}=N /\left(V_{0}^{2} \Delta V\right) .
$$

Ainsi, le rapport $N /\left(V_{0}^{2} \Delta V\right)$ est une constante de l'expérimentation, dans la mesure où $m$ et $\alpha$ restent constants. $A$ est lié uniquement à la sonde et à l'échantillon; $\Gamma(m, \theta)$ est un facteur de correction lié au site $(\theta)$ d'analyse. $A_{\theta}$ apparait donc comme un "rendement effectif » lié au site d'analyse, $A$ est le rendement effectif sur l'axe.

La figure 5 montre une courbe obtenue expérimentalement, donnant le rayon de courbure $R_{0}$ en fonction du potentiel $V_{0}$. $R_{0}$ est calculé à partir de mesures successives de $A_{\theta}$ faites sur des tranches de 200 atomes :

$\frac{V_{0}}{R_{0}}=E \beta=\left|S_{\mathrm{D}} Q \cdot(m+1)^{2} \cdot \Gamma(m, \theta) / L^{2} \cdot v_{\mathrm{at}} \cdot k_{\alpha} \cdot A_{\theta}\right|^{1 / 3}$.

La courbe $R_{0}\left(V_{0}\right)$ montre que $E \beta$ reste sensiblement constant pour un site d'analyse $(\theta)$ donné.

La figure 6 représente la fonction $\Gamma(m, \theta)$ pour $m=1$.

L'influence de $\theta$ sur le "rendement " est d'autant plus grande que l'angle de cône est important. D'autre part la contribution de $m$ sur ces courbes est faible pour $m<2$. 


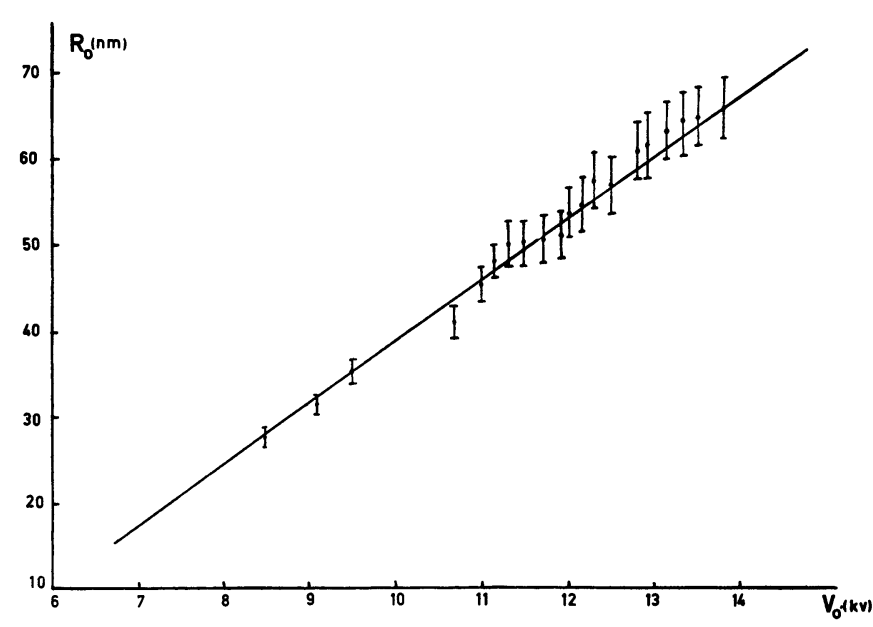

Fig. 5. - Evolution du rayon de courbure de l'échantillon.

[Evolution of the sample curvature radius.]

Le « rendement effectif» $A_{\theta}$ peut être utilisé pour mettre en évidence une évaporation sélective (17). En effectuant une analyse sur un site donné $(\theta=\mathrm{Cte})$ il est possible de montrer expérimentalement une dépendance de $A_{\theta}$ avec la concentration apparente.

Ainsi dans un alliage AB, si une partie des atomes de type $B$ s'évapore au potentiel continu, on observe une concentration $C_{\mathrm{B}}$ inférieure à la concentration nominale $C_{\mathrm{B}}^{0}\left(C_{\mathrm{B}}\right.$ dépend notamment de la valeur de l'impulsion d'évaporation). Si $A_{\theta}$ est le «rendement effectif » en l'absence d'évaporation sélective, le « rendement effectif » apparent est :

$$
A_{\theta}^{\prime}=A_{\theta} \cdot\left(1-C_{\mathrm{B}}^{0}\right) /\left(1-C_{\mathrm{B}}\right) \text {. }
$$

3. Conclusion. - Lorsque l'analyse a lieu en dehors de l'axe de l'échantillon, la direction effective d'analyse n'est plus normale au plan cristallographique étudié, mais fait un angle $\phi$ avec l'axe de la pointe. Cet angle ne dépend que du site d'investigation et de l'angle de cône de l'échantillon. C'est une contrainte supplémentaire dans l'utilisation de la sonde dans l'étude des interfaces et des joints de grain. En particulier les distances analysées doivent être corrigées pour tenir compte de cet effet.

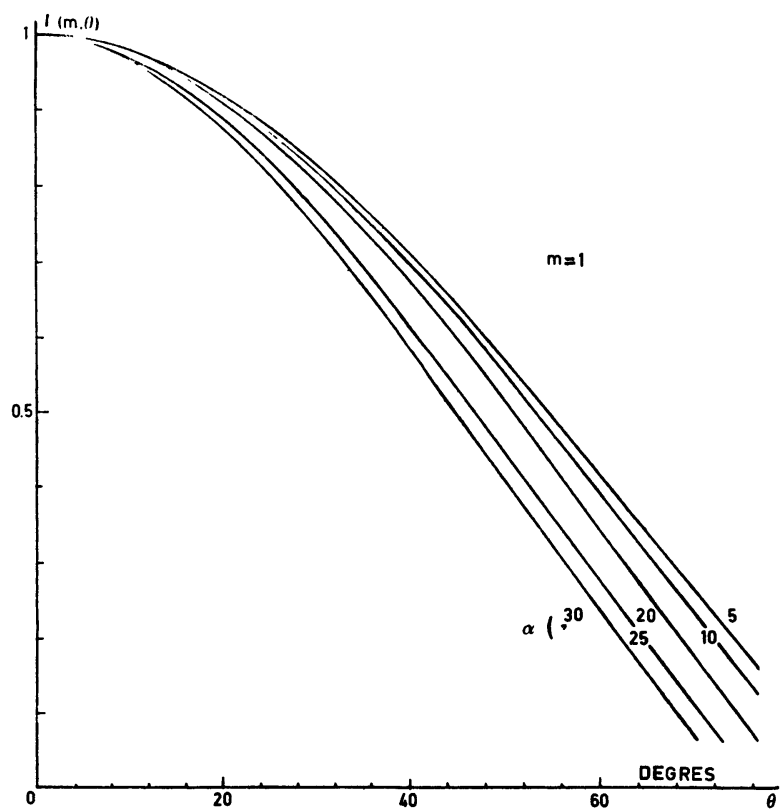

Fig. 6. $-\Gamma(m, \theta)$ pour $m=1$ et pour plusieurs valeurs de l'angle de cône de l'échantillon.

$[\Gamma(m, \theta)$ function for $m=1$ and for various values of the specimen shank angle.]

Les calculs relatifs à la surface d'analyse montrent que les corrections à effectuer sur celle-ci sont faibles tant que l'investigation est effectuée près de l'axe $\left(\theta<30^{\circ}\right)$ et l'expression du volume analysé permet de relier le nombre d'atomes reçus à la profondeur d'analyse.

Enfin, l'étude du " rendement effectif " montre les différentes contributions des fonctions liées à l'appareillage, à l'échantillon, au site d'analyse, à la réception des ions; elle permet de mettre en évidence par exemple une évaporation sélective.

Une meilleure connaissance des phénomènes mis en jeu lors de l'emploi de la sonde atomique permettra d'améliorer l'interprétation des résultats, notamment pour l'étude des interfaces ou de l'ordre à courte distance. 


\section{Bibliographie}

[1] Andren, H. O., Henjered, A., Norden, H., J. Mat. [10] Wei, C. H. Yeu, Seidman, D. N., Mat. Sci. Center Sci. 15 (1980) 2365.

[2] Bach, P. W., Beyer, J., Verbraak, C. A., Script. [11] Goringe, M. J., Smith, D. A., Jernkont Ann. 155 Met. 14 (1980) 205.

[3] Yee, S. Ng, Mclane, S. B., Tsong, J. R. T. T., J. Vac. Sci. Technol. 17 (1980) 154.

[4] Blavette, D., Martin, C., Gallot, J., Script. Met. 16 (1982) 59.

[5] Waugh, A. R., Southon, M. J., Surf. Sci. 89 (1979) 718.

[6] Wagner, R., Brenner, S. S., Acta Metall. 26 (1978) 197.

[7] Wagner, A., Seidman, D. N., J. Nucl. Mat. 83 (1979) 48.

[8] Sarrau, J. M., Gallot, J., Avenel, O., Roubeau, P., J. Phys. E 14 (1981) 800.

[9] Webber, R. D., Smith, R., Walls, J. M., J. Phys. D 12 (1979) 1589. 